\title{
Gravity from Spacetime Thermodynamics
}

\author{
T. Padmanabhan \\ IUCAA, Pune University Campus, Pune 411007 \\ (nabhan@iucaa.ernet.in)
}

\begin{abstract}
The Einstein-Hilbert action (and thus the dynamics of gravity) can be obtained by: (i) combining the principle of equivalence, special relativity and quantum theory in the Rindler frame and (ii) postulating that the horizon area must be proportional to the entropy. This approach uses the local Rindler frame as a natural extension of the local inertial frame, and leads to the interpretation that the gravitational action represents the free energy of the spacetime geometry. As an aside, one obtains an insight into the peculiar structure of Einstein-Hilbert action and a natural explanation to the questions: (i) Why does the covariant action for gravity contain second derivatives of the metric tensor? (ii) Why is the gravitational coupling constant is positive? Some geometrical features of gravitational action are clarified.
\end{abstract}

Keywords: Horizon, Rindler, Entropy, Principle of Equivalence, Holography

\section{Introduction and summary}

The (i) existence of the principle of equivalence and (ii) the connection between gravity and thermodynamics are the two most surprising features of gravity. Among these two, the principle of equivalence finds its natural expression when gravity is described as a manifestation of curved spacetime. This - in turn - makes gravity the only interaction which is capable of wrapping up regions of spacetime so that information from one region is not accessible to observers at another region. Given the fact that entropy of a system is closely related to accessibility of information, it is inevitable that there will be some connection between gravity and thermodynamics (for a review, see references [1] [2]). But, in contrast to the principle of equivalence, years of research in this field (see, for a sample of references [3]), has not led to something more profound or fundamental arising out of this feature.

This suggests that we should learn a lesson from the way Einstein handled the principle of equivalence and apply it in the context of the connection between thermodynamics and gravity. Einstein did not attempt to "derive" principle of equivalence in the conventional sense of the word. Rather, he accepted it as a key feature which must find expression in the way gravity is described - thereby obtaining a geometrical description of gravity. Once the geometrical interpretation of gravity is accepted, it follows that there will arise surfaces which

(c) 2018 Kluwer Academic Publishers. Printed in the Netherlands. 
act as one-way-membranes for information and will thus lead to some connection with thermodynamics. It is, therefore, more in tune with the spirit of Einstein's analysis to accept an inevitable connection between gravity and thermodynamics and ask what such a connection would imply. I will now elaborate this idea further in order to show how powerful it is [4].

The first step in the logic, the principle of equivalence, allows one to define a coordinate system around any event $\mathcal{P}$ in a region of size $L$ (with $L^{2}\left(\partial^{2} g / g\right) \ll 1$ but $L(\partial g / g)$ being arbitrary) in which the spacetime is locally inertial. As the second step, we want to give expression to the fact that there is a deep connection between one-way-membranes arising in a spacetime and thermodynamical entropy. This, of course, is not possible in the local inertial frame since the quantum field theory in that frame, say, does not recognize any non trivial geometry of spacetime. But it is possible to achieve our aim by using a uniformly accelerated frame around $\mathcal{P}$. In fact, around any event $\mathcal{P}$ we have fiducial observers anchored firmly in space with $\mathbf{x}=$ constant and the four-velocity $u^{i}=g_{00}^{-1 / 2}(1,0,0,0)$ and acceleration $a^{i}=u^{j} \nabla_{j} u^{i}$. This allows us to define a second natural coordinate system around any event by using the Fermi-Walker transported coordinates corresponding to these accelerated observers. I shall call this the local Rindler frame. [Operationally, this coordinate system is most easily constructed by first transforming to the locally inertial frame and then using the standard transformations between the inertial coordinates and the Rindler coordinates.] This local Rindler frame will lead to a natural notion of horizon and associated temperature. The key new idea will be to postulate that the horizon in the local Rindler frame also has an entropy per unit transverse area and demand that any description of gravity must have this feature incorporated in it.

What will such a postulate lead to? Incredibly enough, it leads to the correct Einstein-Hilbert action principle for gravity. Note that the original approach of Einstein making use of the principle of equivalence lead only up to the kinematics of gravity — viz., that gravity is described by a curved spacetime with a non trivial metric $g_{a b}$ - and cannot tell us how the dynamics of the spacetime is determined. Taking the next step, using the local Rindler frame and demanding that gravity must incorporate the thermodynamical aspects lead to the action functional itself.

This approach also throws light on (what has been usually considered) a completely different issue: Why does the Einstein-Hilbert action contain second derivatives of the metric tensor? The new approach "builds up" the Einstein-Hilbert action from its surface behaviour and, in this sense, shows that gravity is intrinsically holographic [5]. I use 
this term with the specific meaning that given the form of the action on a two dimensional surface, there is a way of obtaining the full bulk action. In the $(3+1)$ formalism, this leads to the interpretation of the gravitational action as the free energy of spacetime. Einstein's equations are equivalent to the principle of minimization of free energy in thermodynamics.

\section{Gravitational dynamics from spacetime thermodynamics}

The principle of equivalence leads to a geometrical description of gravity in which $g_{a b}$ are the fundamental variables. So we expect the dynamics of gravity to be described by some unknown action functional

$$
A=\int d^{4} x \sqrt{-g} L(g, \partial g) \equiv \int d^{4} x \sqrt{-g} L(g, \Gamma)
$$

involving $g_{a b} \mathrm{~s}$ and their first derivatives $\partial_{c} g_{a b}$ or, equivalently, the set $\left[g_{a b}, \Gamma_{j k}^{i}\right]$ where $\Gamma$ s are the standard Christoffel symbols.

Given any Lagrangian $L(\partial q, q)$ involving only up to the first derivatives of the dynamical variables, it is always possible to construct another Lagrangian $L^{\prime}\left(\partial^{2} q, \partial q, q\right)$, involving second derivatives such that it describes the same dynamics [6]. This idea works for any number of variables (so that $q$ can be a multicomponent entity) dependent on space and time. But I shall illustrate it in the context of point mechanics. The prescription is:

$$
L^{\prime}=L-\frac{d}{d t}\left(q \frac{\partial L}{\partial \dot{q}}\right)
$$

While varying the $L^{\prime}$, one keeps the momenta $(\partial L / \partial \dot{q})$ fixed at the endpoints rather than $q^{\prime}$ s. This is most easily seen by explicit variation; we have

$$
\begin{aligned}
\delta A^{\prime} & =\int_{\mathcal{P}_{1}}^{\mathcal{P}_{2}} d t\left[\frac{\partial L}{\partial q} \delta q+\frac{\partial L}{\partial \dot{q}} \delta \dot{q}\right]-\left.\delta\left(q \frac{\partial L}{\partial \dot{q}}\right)\right|_{\mathcal{P}_{1}} ^{\mathcal{P}_{2}} \\
& =\int_{\mathcal{P}_{1}}^{\mathcal{P}_{2}} d t\left[\frac{\partial L}{\partial q}-\frac{d}{d t}\left(\frac{\partial L}{\partial \dot{q}}\right)\right] \delta q-\left.q \delta p\right|_{\mathcal{P}_{1}} ^{\mathcal{P}_{2}}
\end{aligned}
$$

If we keep $\delta p=0$ at the end points while varying $L^{\prime}$, then we get back the same Euler-Lagrange equations as obtained by varying $L$ and keeping $\delta q=0$ at end points. Since $L=L(\dot{q}, q)$, the quantity $q(\partial L / \partial \dot{q})$ will also depend on $\dot{q}$ and the term $d(q \partial L / \partial \dot{q}) / d t$ will involve $\ddot{q}$. Thus 
$L^{\prime}$ contains second derivatives of $q$ while $L$ contains only up to first derivatives. In spite of the fact that $L^{\prime}$ contains second derivatives of $q$, the equations of motion arising from $L^{\prime}$ are only second order for variation with $\delta p=0$ at end points. It can be shown that, in the path integral formulation of quantum theory, the modified Lagrangian $L^{\prime}$ correctly describes the transition amplitude between states with given momenta (see p. 170 of [6]).

Thus, in the case of gravity, the same equations of motion can be obtained from another (as yet unknown) action:

$$
\begin{aligned}
A^{\prime} & =\int d^{4} x \sqrt{-g} L-\int d^{4} x \partial_{c}\left[g_{a b} \frac{\partial \sqrt{-g} L}{\partial\left(\partial_{c} g_{a b}\right)}\right] \\
& \equiv A-\int d^{4} x \partial_{c}\left(\sqrt{-g} V^{c}\right) \equiv A-\int d^{4} x \partial_{c} P^{c}
\end{aligned}
$$

where $V^{c}$ is made of $g_{a b}$ and $\Gamma_{j k}^{i}$. Further, $V^{c}$ must be linear in the $\Gamma^{\prime}$ s since the original Lagrangian $L$ was quadratic in the first derivatives of the metric. Since $\Gamma$ s vanish in the local inertial frame and the metric reduces to the Lorentzian form, the action $A$ cannot be generally covariant. However, the action $A^{\prime}$ involves the second derivatives of the metric and we shall see later that that the action $A^{\prime}$ is indeed generally covariant.

To obtain a quantity $V^{c}$, which is linear in $\Gamma$ s and having a single index $c$, from $g_{a b}$ and $\Gamma_{j k}^{i}$, we must contract on two of the indices on $\Gamma$ using the metric tensor. (Note that we require $A, A^{\prime}$ etc. to be Lorentz scalars and $P^{c}, V^{c}$ etc. to be vectors under Lorentz transformation.) Hence the most general choice for $V^{c}$ is the linear combination

$$
V^{c}=\left(a_{1} g^{c k} \Gamma_{k m}^{m}+a_{2} g^{i k} \Gamma_{i k}^{c}\right)
$$

where $a_{1}(g)$ and $a_{2}(g)$ are unknown functions of the determinant $g$ of the metric (which is the only (pseudo) scalar entity which can be constructed from $g_{a b} \mathrm{~s}$ and $\left.\Gamma_{j k}^{i} \mathrm{~s}\right)$. Using the identities $\Gamma_{k m}^{m}=\partial_{k}(\ln \sqrt{-g})$, $\sqrt{-g} g^{i k} \Gamma_{i k}^{c}=-\partial_{b}\left(\sqrt{-g} g^{b c}\right)$, we can rewrite the expression for $P^{c} \equiv$ $\sqrt{-g} V^{c}$ as

$$
P^{c}=\sqrt{-g} V^{c}=c_{1}(g) g^{c b} \partial_{b} \sqrt{-g}+c_{2}(g) \sqrt{-g} \partial_{b} g^{b c}
$$

where $c_{1} \equiv a_{1}-a_{2}, c_{2} \equiv-a_{2}$ are two other unknown functions of $g$. If we can fix these coefficients by using a physically well motivated prescription, then we can determine the surface term and by integrating, the Lagrangian $L$. I will now show how this can be done.

Let us consider a static spacetime in which all $g_{a b}$ s are independent of $x^{0}$ and $g_{0 \alpha}=0$. Around any given event $\mathcal{P}$ one can construct a local 
Rindler frame with an acceleration of the observers with $\mathbf{x}=$ constant, given by $a^{i}=(0, \mathbf{a})$ and $\mathbf{a}=\nabla\left(\ln \sqrt{g_{00}}\right)$. This Rindler frame will have a horizon which is a plane surface normal to the direction of acceleration and a temperature $T=|\mathbf{a}| / 2 \pi$ associated with this horizon. I shall postulate that the entropy associated with this horizon is proportional to its area or, more precisely,

$$
\frac{d S}{d A_{\perp}}=\frac{1}{\mathcal{A}_{P}}
$$

where $\mathcal{A}_{P}$ is a fundamental constant with the dimensions of area. It represents the minimum area required to hold unit amount of information and our postulate demands that this number be finite. Given the temperature of the horizon, one can construct a canonical ensemble with this temperature and relate the Euclidean action to the thermodynamic entropy (see, e.g, [2] [7]). Since the Euclidean action can be interpreted as the entropy in the canonical ensemble, I will demand that the surface term in equation (4) should be related to the entropy $S$ by $S=-A_{\text {surface }}$ (with the minus sign arising from standard Euclidean continuation [2]), when evaluated in the local Rindler frame with the temperature $T$. In particular, this result must hold in flat spacetime in Rindler coordinates. [We will see later that the action $A^{\prime}$ is generally covariant and will vanish in the flat spacetime, in the absence of the cosmological constant. It follows that the numerical value of the action $A$ in the Rindler frame is the same as the surface term in equation (4).] In the static Rindler frame, the surface term is

$$
A_{\text {surface }}=\int d^{4} x \partial_{c} P^{c}=\int_{0}^{\beta} d t \int_{\mathcal{V}} d^{3} x \nabla \cdot \mathbf{P}=\beta \int_{\partial \mathcal{V}} d^{2} x_{\perp} \hat{\mathbf{n}} \cdot \mathbf{P}
$$

I have restricted the time integration to an interval $(0, \beta)$ where $\beta=$ $(2 \pi /|\mathbf{a}|)$ is the inverse temperature in the Rindler frame. This is needed since the Euclidean action will be periodic in the imaginary time with the period $\beta$. We shall choose the Rindler frame such that the acceleration is along the $x^{1}=x$ axis. The most general form of the metric representing the Rindler frame can be expressed in the form

$$
\begin{aligned}
d s^{2} & =(1+2 a l) d t^{2}-\frac{d l^{2}}{(1+2 a l)}-\left(d y^{2}+d z^{2}\right) \\
& =[1+2 a l(x)] d t^{2}-\frac{l^{\prime 2}}{[1+2 a l(x)]} d x^{2}-\left(d y^{2}+d z^{2}\right)
\end{aligned}
$$

where $l(x)$ is an arbitrary function and $l^{\prime} \equiv(d l / d x)$. [Since the acceleration is along the $\mathrm{x}$-axis, the metric in the transverse direction is unaffected. The first form of the metric is the standard Rindler frame 
in the $(t, l, y, z)$ coordinates. We can, however, make any coordinate transformation from $l$ to some other variable $x$ without affecting the planar symmetry or the static nature of the metric. This leads to the general form of the metric given in the second line, in terms of the $(t, x, y, z)$ coordinates.] Evaluating the surface term $P^{c}$ in (6) for this metric, we get the only non zero component to be

$$
P^{x}=-2 a c_{2}(g)-[1+2 a l(x)] \frac{l^{\prime \prime}}{l^{\prime 2}}\left[c_{1}(g)-2 c_{2}(g)\right]
$$

so that the action in (8) becomes

$$
A=\beta P^{x} \int d^{2} x_{\perp}=\beta P^{x} A_{\perp}=-S
$$

where $A_{\perp}$ is the transverse area of the $(y-z)$ plane. The last equality identifies the entropy $S$, which is equal the Euclidean action, with the minus sign arising from standard Euclidean continuation. From our postulate (7) it follows that

$$
\frac{d S}{d A_{\perp}}=2 a \beta c_{2}(g)+\beta\left[c_{1}-2 c_{2}\right](1+2 a l) \frac{l^{\prime \prime}}{l^{\prime 2}}=\frac{1}{\mathcal{A}_{P}}
$$

For this quantity to be a constant independent of $x$ for any choice of $l(x)$, the second term must vanish requiring $c_{1}(g)=2 c_{2}(g)$. An explicit way of obtaining this result is to consider a class of functions $l(x)$ which satisfy the relation $l^{\prime}=(1+2 a l)^{n}$ with $0 \leq n \leq 1$. Then

$$
\beta\left[c_{1}\left(l^{\prime}\right)-2 c_{2}\left(l^{\prime}\right)\right](1+2 a l) \frac{l^{\prime \prime}}{l^{\prime 2}}=2 a \beta\left[c_{1}\left(l^{\prime}\right)-2 c_{2}\left(l^{\prime}\right)\right] n
$$

which can be independent of $n$ and $x$ only if $c_{1}(g)=2 c_{2}(g)$. Further, using $a \beta=2 \pi$, we find that $c_{2}(g)=\left(4 \pi \mathcal{A}_{p}\right)^{-1}$ which is a constant independent of $g$. Hence $P^{c}$ has the form

$$
\begin{aligned}
P^{c} & =\frac{1}{4 \pi \mathcal{A}_{P}}\left(2 g^{c b} \partial_{b} \sqrt{-g}+\sqrt{-g} \partial_{b} g^{b c}\right)=\frac{\sqrt{-g}}{4 \pi \mathcal{A}_{P}}\left(g^{c k} \Gamma_{k m}^{m}-g^{i k} \Gamma_{i k}^{c}\right) \\
& =-\frac{1}{4 \pi \mathcal{A}_{P}} \frac{1}{\sqrt{-g}} \partial_{b}\left(g g^{b c}\right)
\end{aligned}
$$

The second equality is obtained by using the standard identities mentioned after equation (5) while the third equality follows directly by combining the two terms in the first expression. This result is remarkable and let me discuss it before proceeding further.

The general form of $P^{c}$ which we obtained in (6) is not of any use unless we can fix $\left(c_{1}, c_{2}\right)$. For static configurations, we can convert the 
extra term to an integral over time and a two-dimensional spatial surface. This is true for any system, but in general, the result will not have any simple form and will involve an undetermined range of integration over time coordinate. But in the case of gravity, two natural features conspire together to give an elegant form to this surface term. First is the fact that Rindler frame has a periodicity in Euclidean time and the range of integration over the time coordinate is naturally restricted to the interval $(0, \beta)=(0,2 \pi / a)$. The second is the fact that the surviving term in the integrand $P^{c}$ is linear in the acceleration $a$ thereby neatly canceling with the $(1 / a)$ factor arising from time integration. [I will discuss these features more in section (3).]

Given the form of $P^{c}$ we need to solve the equation

$$
\left(\frac{\partial \sqrt{-g} L}{\partial g_{a b, c}} g_{a b}\right)=P^{c}=\frac{1}{4 \pi \mathcal{A}_{P}}\left(2 g^{c b} \partial_{b} \sqrt{-g}+\sqrt{-g} \partial_{b} g^{c b}\right)
$$

to obtain the first order Lagrangian density. It is straightforward to show [6] that this equation is satisfied by the Lagrangian

$$
\sqrt{-g} L=\frac{1}{4 \pi \mathcal{A}_{P}}\left(\sqrt{-g} g^{i k}\left(\Gamma_{i \ell}^{m} \Gamma_{k m}^{\ell}-\Gamma_{i k}^{\ell} \Gamma_{\ell m}^{m}\right)\right) .
$$

This is the second surprise. The Lagrangian which we have obtained is precisely the first order Dirac-Schrodinger Lagrangian for gravity (usually called the $\Gamma^{2}$ Lagrangian). Note that we have obtained it without introducing the curvature tensor anywhere in the picture. Once again, this is unlikely to be a mere accident.

Given the two pieces, the final second order Lagrangian follows from our equation (4) and is, of course, the standard Einstein-Hilbert Lagrangian.

$$
\sqrt{-g} L_{\text {grav }}=\sqrt{-g} L-\frac{\partial P^{c}}{\partial x^{c}}=\left(\frac{1}{4 \pi \mathcal{A}_{P}}\right) R \sqrt{-g} .
$$

Thus our full second order Lagrangian turns out to be the standard Einstein-Hilbert Lagrangian. We have obtained this result by just postulating that the surface term in the action should be proportional to the entropy per unit area. This postulate uniquely determines the gravitational action principle and gives rise to a generally covariant action. The surface terms dictate the form of the Einstein Lagrangian in the bulk. The idea that surface areas encode bits of information per quantum of area allows one to determine the nature of gravitational interaction on the bulk, which is an interesting realization of the holographic principle.

I stress the fact that there is a very peculiar identity connecting the $\Gamma^{2}$ Lagrangian $L$ and the Einstein-Hilbert Lagrangian $L_{\text {grav }}$, encoded 
in equation (17). This relation, which is purely a differential geometric identity, can be stated through the equations:

$$
L_{\text {grav }}=L-\nabla_{c}\left[g_{a b} \frac{\partial L}{\partial\left(\partial_{c} g_{a b}\right)}\right] ; \quad L=L_{\text {grav }}-\nabla_{c}\left[\Gamma_{a b}^{j} \frac{\partial L_{\text {grav }}}{\partial\left(\partial_{c} \Gamma_{a b}^{j}\right)}\right]
$$

This relationship between the three terms defies any simple explanation in conventional approaches to gravity but arises very naturally in the approach presented here.

The solution to (15) obtained in (16) is not unique. However, self consistency requires that the final equations of motion for gravity must admit the line element in (9) as a solution. It can be shown. by fairly detailed algebra, that this condition makes the Lagrangian in (15) to be the only solution. In particular, since we are demanding the flat spacetime to be a solution to the field equations, the cosmological constant in the pure gravity sector must be zero. [This, of course, does not prevent a cosmological constant arising from the matter sector of the theory.]

\section{Structure of Einstein-Hilbert action}

The approach leads to new insights regarding the peculiar structure of Einstein-Hilbert and the $(3+1)$ formalism of gravity. To discuss these features, it is convenient to temporarily switch to the signature $(-+++)$ so that the spatial metric is positive definite. We will foliate the spacetime by a series of space like hyper-surfaces $\Sigma$ with $u^{i}$ as normal; then $g^{i k}=h^{i k}-u^{i} u^{k}$ where $h^{i k}$ is the induced metric on $\Sigma$. From the covariant derivative $\nabla_{i} u_{j}$ of the normals to $\Sigma$, one can construct only three vectors $\left(u^{j} \nabla_{j} u^{i}, u^{j} \nabla^{i} u_{j}, u^{i} \nabla^{j} u_{j}\right)$ which are linear in covariant derivative operator. The first one is the acceleration $a^{i}=$ $u^{j} \nabla_{j} u^{i}$; the second identically vanishes since $u^{j}$ has unit norm; the third, $u^{i} K$, is proportional to the trace of the extrinsic curvature $K=$ $-\nabla^{j} u_{j}$ of $\Sigma$. Thus $V^{i}$ in the surface term in equation (4) must be a linear combination of $u^{i} K$ and $a^{i}$. In fact, one can show that (see, e.g. equation (21.88) of the first reference in [8])

$$
R={ }^{3} \mathcal{R}+K_{a b} K^{a b}-K_{a}^{a} K_{b}^{b}-2 \nabla_{i}\left(K u^{i}+a^{i}\right) \equiv \mathcal{L}-2 \nabla_{i}\left(K u^{i}+a^{i}\right)
$$

where $\mathcal{L}$ is the ADM Lagrangian. To prove this, we begin with the relation

$$
R=-R g_{a b} u^{a} u^{b}=2\left(G_{a b}-R_{a b}\right) u^{a} u^{b}
$$

and rewrite the first term using the identity:

$$
2 G_{a b} u^{a} u^{b}={ }^{3} \mathcal{R}-K_{a b} K^{a b}+K_{a}^{a} K_{b}^{b}
$$


As for the second term in (20), we note that $R_{a b c d} u^{d}=\left(\nabla_{a} \nabla_{b} u_{c}-\right.$ $\left.\nabla_{b} \nabla_{a} u_{c}\right)$ giving

$$
\begin{aligned}
R_{b d} u^{b} u^{d} & =g^{a c} u^{b} u^{d} R_{a b c d}=\left(u^{b} \nabla_{a} \nabla_{b} u^{a}-u^{b} \nabla_{b} \nabla_{a} u^{a}\right) \\
& =\nabla_{a}\left(u^{b} \nabla_{b} u^{a}\right)-\left(\nabla_{a} u^{b}\right)\left(\nabla_{b} u^{a}\right)-\nabla_{b}\left(u^{b} \nabla_{a} u^{a}\right)+\left(\nabla_{b} u^{b}\right)^{2} \\
& =\nabla_{i}\left(K u^{i}+a^{i}\right)-K_{a b} K^{a b}+K_{a}^{a} K_{b}^{b}
\end{aligned}
$$

Using (22) and (21) we can rewrite (20) in the form of (19).

Let us now use (19) to integrate $(R / 16 \pi)$ over a four volume $\mathcal{V}$ bounded by two space-like surfaces $\Sigma_{1}$ and $\Sigma_{2}$ (with normals $u^{i}$ ) and two time-like surfaces $\mathcal{S}_{1}$ and $\mathcal{S}_{2}$ (with normals $n^{i}$ ). The induced metric on the space-like surface $\Sigma$ is $h_{a b}=g_{a b}+u_{a} u_{b}$ while the metric on the time-like surface $\mathcal{S}$ is $\gamma_{a b}=g_{a b}-n_{a} n_{b}$. These two surfaces will intersect on a two-dimensional surface $\mathcal{Q}$ on which the metric is $\sigma_{a b}=$ $h_{a b}-n_{a} n_{b}=g_{a b}+u_{a} u_{b}-n_{a} n_{b}$. Integrating both sides of (19) over $\mathcal{V}$ we now get

$$
\begin{gathered}
A_{\mathrm{EH}}=\frac{1}{16 \pi} \int_{\mathcal{V}} R \sqrt{-g} d^{4} x=\frac{1}{16 \pi} \int_{\mathcal{V}} \mathcal{L} \sqrt{-g} d^{4} x-\frac{1}{8 \pi} \int_{\Sigma_{1}}^{\Sigma_{2}} K \sqrt{h} d^{3} x \\
-\frac{1}{8 \pi} \int_{\mathcal{S}_{1}}^{\mathcal{S}_{2}}\left(a_{i} n^{i}\right) \sqrt{\sigma} d^{2} x N d t
\end{gathered}
$$

where $g_{00}=-N^{2}$. In a static spacetime with a horizon: (i) $K=0$ making the second term on the right hand side vanish. (ii) The integration over $t$ becomes multiplication by $\beta$. (iii) Further, as the surface $\mathcal{S}_{1}$ approaches the horizon, the quantity $N\left(a_{i} n^{i}\right)$ tends to $(-\kappa)$ where $\kappa$ is the surface gravity of the horizon, which is constant over the horizon. Using $\beta \kappa=2 \pi$, the last term gives, on the horizon, the contribution

$$
\frac{\kappa}{8 \pi} \int_{0}^{\beta} d t \int d^{2} x \sqrt{\sigma}=\frac{1}{4} \mathcal{A}_{H}
$$

where $\mathcal{A}_{H}$ is the area of the horizon. In the Euclidean sector the first term gives $\beta E$ where $E$ is the integral of the ADM Hamiltonian over the spatial volume. We thus get the result

$$
A_{\mathrm{EH}}^{\text {Euclidian }}=\frac{1}{4} \mathcal{A}_{H}-\beta E=(S-\beta E)
$$

which is the free energy. For any static spacetime geometry, having a periodicity $\beta$ in the Euclidean time, the Euclidean gravitational action represents the free energy of the spacetime; the first order term gives the Hamiltonian and the surface term gives the entropy.

More generally, the analysis suggests a remarkably simple, thermodynamical, interpretation of semiclassical gravity. In any static spacetime with a metric

$$
d s^{2}=N^{2}(\mathbf{x}) d t^{2}-\gamma_{\alpha \beta}(\mathbf{x}) d x^{\alpha} d x^{\beta}
$$


we have $R={ }^{3} R+2 \nabla_{i} a^{i}$ where $a_{i}=\left(0, \partial_{\alpha} N / N\right)$ is the acceleration of $\mathbf{x}=$ constant world lines. Then, limiting the time integration to $(0, \beta)$, say, the Einstein-Hilbert action becomes

$$
A=\frac{\beta}{16 \pi} \int_{\mathcal{V}} d^{3} x N \sqrt{\gamma}^{3} R+\frac{\beta}{8 \pi} \int_{\partial \mathcal{V}}\left(a^{\alpha} n_{\alpha}\right) d^{2} \mathcal{S} \equiv \beta E-S
$$

where the first term is proportional to energy (in the sense of spatial integral of ADM Hamiltonian) and the second term is proportional to entropy in the presence of horizon. The variation of this action - which leads to Einstein's equation - is equivalent to the thermodynamic identity. (This result is explored in detail for spherically symmetric spacetimes in [7]).

The surfaces $\Sigma, \mathcal{S}$ as well as the two surface $\mathcal{Q}$ on which they intersect will have corresponding extrinsic curvatures $K_{a b}, \Theta_{a b}$ and $q_{a b}$. In the literature, it is conventional to write the Einstein-Hilbert action as a term having only the first derivatives, plus an integral over the trace of the extrinsic curvature of the bounding surfaces. It is easy to obtain this form using the foliation condition $n^{i} u_{i}=0$ between the surfaces and noting:

$$
n_{i} a^{i}=n_{i} u^{j} \nabla_{j} u^{i}=-u^{j} u^{i} \nabla_{j} n_{i}=\left(g^{i j}-h^{i j}\right) \nabla_{j} n_{i}=-(\Theta-q)
$$

where $\Theta \equiv \Theta_{a}^{a}$ and $q \equiv q_{a}^{a}$ are the traces of the extrinsic curvature of the 2-surface when treated as embedded in the 4-dimensional or 3dimensional enveloping manifolds. Using $(28)$ to replace $\left(a_{i} n^{i}\right)$ in the last term of (23), we get the result

$$
\begin{aligned}
A_{\mathrm{EH}} & +\frac{1}{8 \pi} \int_{\Sigma_{1}}^{\Sigma_{2}} K \sqrt{h} d^{3} x-\frac{1}{8 \pi} \int_{\mathcal{S}_{1}}^{\mathcal{S}_{2}} \Theta \sqrt{\sigma} d^{2} x N d t \\
& =\frac{1}{16 \pi} \int_{\mathcal{V}} \mathcal{L} \sqrt{-g} d^{4} x-\frac{1}{8 \pi} \int_{\mathcal{S}_{1}}^{\mathcal{S}_{2}} q \sqrt{\sigma} d^{2} x N d t
\end{aligned}
$$

In the first term on the right hand side, the ADM Lagrangian $\mathcal{L}$ contains ${ }^{3} \mathcal{R}$ which in turn involves the second derivatives of the metric tensor. The second term on the right hand side removes these second derivatives making the right hand side equal to the quadratic $\Gamma^{2}$ action for gravity. On the left hand side, the second and third terms are the integrals of the extrinsic curvatures over the boundary surfaces which, when added to the Einstein-Hilbert action gives the quadratic action without second derivatives. (This is the standard result often used in the literature). Unfortunately, this form replaces the normal component of the acceleration $a^{i} n_{i}$ in $(23)$ by $(\Theta-q)$ and combines $q$ with ${ }^{3} \mathcal{R}$ to get the first order Lagrangian. In the process, the normal component 
of the acceleration disappears and we miss the nice interpretation of Einstein-Hilbert action as the free energy of spacetime.

\section{Conclusions}

The approach adopted here is a natural extension of the original philosophy of Einstein; viz., to use non inertial frames judiciously to understand the behaviour of gravity. In the original approach, Einstein used the principle of equivalence which leads naturally to the description of gravity in terms of the metric tensor. Unfortunately, classical principle of equivalence cannot take us any further since it does not encode information about the curvature of spacetime. However, the true world is quantum mechanical and one would like to pursue the analogy between non inertial frames and gravitational field into the quantum domain. Here the local Rindler frame arises as the natural extension of the local inertial frame and the study of the thermodynamics of the horizon shows a way of combining special relativity, quantum theory and physics in the non inertial frame. I have shown that these components are adequate to determine the action functional for gravity and, in fact, leads to the Einstein-Hilbert action. This is remarkable because we did not introduce the curvature of spacetime explicitly into the discussion and - in fact - the analysis was done in a Rindler frame which is just flat spacetime. The idea works because the action for gravity splits up into two natural parts neither of which is generally covariant but are related to each other by the remarkable identity (18) which — as far as I know - was not noticed before. The sum of the two parts is generally covariant but the expression for individual parts can be ascertained in the local Rindler frame specifically because these parts are not generally covariant.

The fundamental postulate we use is in equation (7) and it does not refer to any horizon. To see how this comes about, consider any spatial plane, say the $y-z$ plane, in flat spacetime. It is always possible to find a Rindler frame in the flat spacetime such that the chosen surface acts as the horizon for some Rindler observer. In this sense, any plane in flat spacetime must have an entropy per unit area. Microscopically, I would expect this to arise because of the entanglement over length scales of the order of $\sqrt{\mathcal{A}_{P}}$. We have defined in (7) the entropy per unit area rather than the total entropy in order to avoid having to deal with global nature of the surfaces (whether the surface is compact, non compact etc.). This approach also provides a natural explanation as to why the gravitational coupling constant is positive. It is positive because entropy and area are positive quantities. 
The result emphasizes the role of two dimensional surfaces in fundamental physics. A two dimensional surface is the basic minimum one needs to produce region of inaccessibility and thus entropy from lack of information. When one connects up gravity with spacetime entropy it is is inevitable that the coupling constant for gravity has the dimensions of area in natural units. The next step in such an approach will be to find the fundamental units by which spacetime areas are made of and provide a theoretical, quantum mechanical description for the same. This will lead to the proper quantum description of spacetime with Einstein action playing the role of the free energy in the thermodynamic limit of the spacetime.

\section{Acknowledgement}

I thank Apoorva Patel and K. Subramanian for several useful discussions. I thank the organizers of the "Fred Hoyle's Universe" conference (Cardiff, June, 2002) for inviting me to give the talk and providing local hospitality.

\section{References}

1. Birrell N.D and Davies P.C.W, Quantum fields in curved space, (Cambridge University Press, Cambridge, 1982).

2. Padmanabhan T., Mod.Phys.Letts. A 17, 923 (2002). [gr-qc/0202078].

3. Bekenstein, J.D., Phys. Rev. D 7, 2333 (1973); Hawking S.W., Comm.Math.Phys., 43, 199 (1975) Gerlach, U.H., Phys. Rev. D 15, 1479 (1976); t'Hooft, G., Nucl. Phys. B256, 727 (1985). York, J., Phys. Rev. D 15, 2929 (1985); Zurek, W.H. and Thorne, K.S., Phys. Rev. lett. 54, 2171 (1985); Bombelli. L et al., , Phys. Rev. D 34, 3, 73 (1986); For an earlier attempt, similar in spirit to the current paper, see Jacobson, T. Phys.Rev.Letts., 75, 1260 (1995).

4. This idea was suggested in T. Padmanabhan, The Holography of gravity encoded in a relation between Entropy, Horizon Area and the Action for gravity [Second Prize essay; Gravity Research Foundation Essay Contest, 2002] and elaborated in T. Padmanabhan, Mod.Phys.Letts. A , 17, 1147 (2002) [hep-th/0205278].

5. 't Hooft, G Dimensional Reduction in quantum gravity, gr-qc/9310026; The holographic principle, hep-th/0003004; L. Susskind (1995) J.Math.Phys., 36, 6377; for a recent review, see R. Bousso,, The holographic principle, hepth/0203101.

6. Lynden-Bell D. and T. Padmanabhan, (1994), unpublished; Padmanabhan,T., Cosmology and Astrophysics - through problems (Cambridge university press, 1996) p. 170 ; p. 325.

7. Padmanabhan T., Class.Quan.Grav. (2002), 19, 5387; [gr-qc/0204019].

8. Miser, C.W., Thorne K.S., Wheeler, J.A., Gravitation (Freeman and co., 1973), p.520; York,J.W (1987) in Between Quantum and Cosmos, eds W.H.Zurek et al (Princeton University Press, Princeton, 1988), p.246. 